\title{
Is urbanization a risk factor for substance misuse?
}

\section{Fabrizio Schifano}

$(* *)$ University of Hertfordshire, Pharmacy and Postgraduate Medical Schools, Hatfield, Herts, AL10 9AB (UK).

Address for correspondence:

Professor F Schifano

Chair in Clinical Pharmacology and Therapeutics Associate Dean, Postgraduate Medical School Hon Consultant Psychiatrist (Addictions)

University of Hertfordshire School of Pharmacy Hatfield, Herts AL10 9AB (UK) telephone: +44 (0)1707 283107 fax: +44 (0)1707 284506 mobile: +44 (0)778900 6809 email: F.Schifano@herts.ac.uk 


\section{Abstract}

Purpose of the review: More than half of the world population is living in urban areas, but the evidence about urban-rural differences in drug use is not clearly defined. The present paper aims at reviewing the international literature addressing the role of urbanization and related parameters in association with substance misuse. In particular, the evidence of urbanization being a risk factor for substance misuse will be here examined.

Recent findings: Although it has been suggested that substance misuse is typically a characteristic of large urban areas, this did not appear to be a consistent observation from the present literature review. Furthermore, the urban context may offer a relative proximity of health services and prompter emergency intervention in case of overdose.

Summary: Although more efforts have to be put into explaining substance misuse in general, identifying which characteristics of the urban context, and under which circumstances, are modifiable, is an important theoretical, empirical, and public health question

Key words: urbanization; drug misuse; drug addiction; substance misuse; risk factors; drugs; urban density; homelessness.

\section{Introduction}

It has been reported (1) that by now more than half of the world population is living in urban areas, with some 400 cities having a population in excess of a million people. Urbanization, a phenomenon which has become increasingly important over the last 100 years or so, has been defined as the process of becoming urban, and it reflects aggregate population growth in cities (2), be it through either natural population increase or migration. Therefore, the urban environment becomes important as a potential determinant of both health and health behaviour (1). Cities change over time, and this may influence as well the different health population index parameters. For example, suburbanization (or movement of people from city centre to surrounding areas; 2 ) led to dramatic modifications in population resources in many cities. One could note that heroin, crack and HIV infection first spread in metropolitan sub-populations in the 1970s and 1980s (2) before being disseminated throughout the whole index country. Substance misuse may be affected as well by changes in urban living conditions. Homelessness, as an example of the importance of markets to the health of urban populations, has indeed been frequently associated with a variety of adverse health outcomes, including drug and alcohol misuse (3). It has recently been confirmed that psychiatric disorders are more common and more 
complex in more urbanized areas (4). Similarly, one could conceptualize substance misuse as an urban problem, in contrast with the 'healthy' rural life-style. In line with this, the present paper aims at reviewing the international literature addressing the role of urbanization and related parameters in association with substance misuse. In particular, the evidence of urbanization being a risk factor for substance misuse will be here examined.

\section{Literature search methods}

The papers here commented were identified with the help of searching databases, including Pubmed (http://www.ncbi.nlm.nih.gov/sites/entrez) and ISI web of knowledge (http://portal.isiknowledge.com/). No studies were excluded due to being written in a language other than English. The literature search was carried out using a combination of the following keywords: urbanization; substance misuse; drug misuse; risk factors; drugs; urban density; homelessness. Articles eligible for inclusion were either review or research papers published between 1965 and March 2008, with particular attention to those papers published over the last 3 years. References of all retrieved articles were examined for additional studies. In total, some 80 articles were analyzed, and for 32 of them the focus was felt to be of interest for the present review.

\section{Substance misuse and urbanization issues}

Theoretically, the most important factors that affect health in general can be considered within three broad themes: the social environment, the physical environment, and access to health and social services (5). Similarly, the different characteristics of the urban environment that may be associated with substance misuse may be conceptualized in a framework which has three components (1). First, urban characteristics, being further sub-classified in primary (i.e.: determinants of the infrastructure, employment, local educational and health resources) and secondary (mediating the relation between the primary neighbourhood factors and the population drug use) determinants. Second, social networks and psychosocial stressors; they moderate the association between characteristics of the urban environment and substance misuse. Third, area characteristics and living condition; substance misuse may be in fact conceived as a result not only of the psychobiological profile of the index user, but also of the characteristics of the user's area, which include income distribution levels.

Urbanization, stress, maladaptation and substance misuse

The stress resulting from living in high income disparity/disadvantaged urban areas with chronic exposure to threatening conditions may be associated with inter-individual 
tension and likelihood of violence, which in turn may result in increasing levels of substance misuse (1). Furthermore, people who live in economically or racially segregated communities may have disproportionate exposure, susceptibility and response to economic and social deprivation, toxic substances and hazardous conditions (1). Psychoactive substances may be used to relieve stress, and stress-related substance use may contribute to abuse and dependence. In other words, one could conceive drug use in disadvantaged and segregated urban neighbourhoods as a coping mechanism in response to a number of stressful life experiences (1). In contrast with this, however, Jiang (6) carried out an epidemiological survey in the residential areas of 6 neighbourhood committees of the Chao Yang District of Beijing in search of the prevalent status and related factors of drug abuse among 1,822 households out of 6,114 population. In exploring the causation of drug abuse, it was found that family structure, economic status, individual social function or stressful life events were not correlated and the Authors concluded that their findings did not support the assumption that drug use is a coping method to environmental maladaptation.

Joutsenniemi (7) analyzed data from a nationally representative cross-sectional survey conducted in Finland in 2000-2001 ( $\mathrm{N}=$ 4589 in the age-range of 30-54 years, response rate $81 \%$ ). Among both genders, adjusting for main activity and financial difficulties attenuated the odds for heavy drinking and alcohol dependence by approximately $5-30 \%$ each. Furthermore, among women, adjusting for urbanization attenuated the odds for heavy drinking by approximately $15-45 \%$. They concluded that unemployment, financial difficulties and low social support, and among women also living in an urban area, seem to contribute to the excess risk.

Madsen et al (8) analysed the effects of migration and urbanization on alcohol intake among a population of Greenland Inuit with the means of a population-based cross-sectional study of 4,139 Inuit randomly selected from Denmark and four areas of western Greenland. Data collection was based on interviews and selfadministered questionnaires. They found that the population living in Denmark had a higher mean alcohol intake than those living in Greenland. In contrast, a higher proportion of individuals with episodes of binge drinking was observed in both large and small communities in Greenland. When comparing Inuit living in Denmark according to length of stay in Denmark, the Authors found a significantly increase in prevalence of binge drinking with length of stay, while no significant variation with length of stay. Madsen et al (8) concluded that it was unclear if the observed differences in the alcohol intake among Inuit living in Denmark and in Greenland respectively were to be attributed either to urbanization or to migration. 
Urbanization, neighbourhood disadvantage and availability of health/social services as a mitigating factor for substance misuse

The relation between neighbourhood disadvantage and risk behaviour may be more pronounced in the absence of formal social/health services. In urban areas with more available social and health services, the relation between a deteriorating built environment and drug misuse and their consequences may be attenuated (1). In confirming this, March et al (9), with the means of a cross-sectional survey, described the characteristics seen among socially excluded drug users in 10 cities from 9 European countries, and aimed at identifying which social exclusion indicators (i.e. housing, employment, education) were most closely linked to intravenous drug use. The sample included 1,879 participants who had used heroin and/or cocaine over the previous year. Cannabis, heroin and cocaine turned out to be the most widely used substances; $14.2 \%$ of the interviewees were homeless; $11.3 \%$ had a regular job, and $35.2 \%$ were involved in illegal activities. They concluded that social exclusion variables associated with intravenous drug use were incarceration, homelessness, irregular employment, and delinquency.

Furthermore, Day et al (10) aimed at comparing: patterns of drug use, harms, and service access and utilisation among rural and metropolitan injecting drug users (IDU). They interviewed 164 rural and 96 metropolitan IDU from seven different New South Wales Area Health Services, recruited through needle and syringe programs (NSPs), snowballing techniques and advertisement. Age, gender, education and employment were similar for rural and metropolitan participants. Both samples reported use of a range of drugs, but rural participants were less likely than metropolitan participants to report daily heroin use ( $2 \%$ vs $10 \%)$, but more likely to report having injected morphine (50\% vs $21 \%$ ) in the last six months. Rural participants were less likely to report use of NSPs ( $36 \%$ vs $80 \%$ ) and reported a number of barriers to NSP access and also to drug treatment services. Rural participants reported a significantly longer period of time between blood-borne virus testing. The Authors concluded that samples of rural IDU were similar to metropolitan, although some differences in patterns of drug use were identified. However, service provision, including access to new injecting equipment, blood-borne virus testing and drug treatment was found to cause considerable problems for rural IDU.

In the context of substance use and misuse, availability of regular, good quality, medical care may contribute to lower prevalence of drug use in urban areas. Greacen et al (11) focussed their research study on Paris, where high rates of generalised anxiety, drug and alcohol abuse/dependence and greater use of psychoactive 
medication are reported. Although the Greater Paris area has a much higher density of GPs and specialists than the national mean, there are considerable variations within the region itself, with the central area having up to four times as many GPs or psychiatrists as the outer suburbs. Although number of people receiving mental health care has been rising dramatically over the last 15 years, Paris has considerably less adult psychiatry beds and day care places per head of population than the rest of France. Current planning targets in Paris include a more equitable distribution of mental health care service provision for the rapidly evolving urban population, and early prevention of suicide and drug and alcohol misuse.

From the UK, Squires et al (12) aimed at determining the prevalence and distribution of opiate and cocaine misuse in the City of Liverpool in 1991. Data on 1427 individuals were analysed; in the 15-29 year age group, the prevalence of drug abuse was 16.9 per 1000; Drug Dependency Unit attenders were 7.2 times less likely to be arrested for possession than non-attenders. There was a strong correlation between the distribution of known drug use and material deprivation, as measured by the Townsend index $(r=0.75 ; p<$ 0.001 ). The Authors suggested that people in contact with drug services were less likely to commit crimes of possession of class $A$ drugs than those not in contact with drug services. Furthermore, they suggested that the purchasing of services for drug misusers should be focused on areas of deprivation.

Furthermore, Friedman et al (13) aimed at determining which US metropolitan area characteristics were associated with drug treatment coverage. They carried out a secondary analysis of official data, including the number of injection drug users in treatment and other variables, for 94 large US metropolitan areas and estimated the number of injection drug users in these areas. Independent predictors of higher drug treatment coverage for injectors included: presence of organisations that support treatment; education expenditures per capita in each metropolitan area. They concluded that the presence of organisations that support drug treatment may be important determinants of treatment coverage for injectors.

Level of population density as a risk factor for substance misuse, associated hospitalizations and related fatalities

An obvious risk factor of living in a large city may be given by the population density. In fact, one could think that more densely populated urban neighbourhoods are more likely to present with illicit sales of substances. Traditionally, there are two identifiable illicit drug markets $(14,15)$. The 'street market' is particularly represented in large metropolitan areas and usually provides heroin and crack cocaine; it is run by hierarchical organized crime, using mobile phone communications, with all participants controlled by 
the threat of actual violence. Conversely, the 'free market' involves the selling of cannabis and ecstasy amongst small groups of friends (16).

The relationship between population density and drug use risk behaviour may be mediated by the presence of social networks that encourage drug use and misuse (1). On the other hand, apart from socializing with drug users, the availability of drugs is clearly among the necessary conditions for an outbreak of drug misuse in a particular community, and this is likely to be more important than the presence of predisposed individuals (17).

Sundquist and Frank (18) analysed whether the level of urbanization was associated with hospital admissions for alcohol and drug abuse, after adjustment for individual demographic and socioeconomic characteristics. Their 1996-1999 follow-up study regarded the entire 4.5 million Swedish adult population. They measured hospital admission rates for alcohol abuse $(12,812$ events) and drug abuse (6459 events). Their results showed that with increasing level of urbanization the hospital admission rates for alcohol abuse and drug abuse increased sharply. After adjustment for a number of parameters, the hazard ratios for hospital admission for alcohol abuse were 1.76 [confidence interval $(\mathrm{CI})=1.58-1.96$ ] for women and $1.71(\mathrm{CI}=1.60-1.82)$ for men in the most densely populated areas. For drug abuse the corresponding hazard ratios were 1.89 $(\mathrm{CI}=1.67-2.15)$ for women and $2.38(\mathrm{CI}=2.12-2.67)$ for men. They concluded that a high level of urbanization was associated with increased hospital admission rates for alcohol and drug abuse.

Shah and Landen (19) examined the relationship between the types of drugs causing poisoning deaths and the levels of urbanization in analyzing the data provided by the New Mexico Office of the Medical Investigator for 1994-2003. All counties in New Mexico were classified as metropolitan or micropolitan statistical areas. They found that deaths from illicit-drug poisoning were twice as likely to occur in metropolitan areas as nonmetropolitan areas. However, deaths from prescription-drug poisoning were most likely to occur in micropolitan areas.

Most recent findings from the UK National Programme on Substance Abuse Deaths (np-SAD; 20) seem to contradict the assumption that most feared consequences of drug consumption, e.g. drug-related fatalities levels, may be higher in high density population areas. Notifications of 1,366 drug-related deaths occurring in 2006 were received by some $90 \%$ of coroners' jurisdictions in England and Wales. The majority (76\%) of the cases were male, under 45 years. $(71 \%)$ and white $(95.5 \%)$. Most died from either accidental $(60.5 \%)$ or intentional $(13.1 \%)$ poisoning. In most cases, the principal substances implicated were heroin/morphine (46\%), alcohol in combination with other substances $(32 \%)$, other opiates/opioid analgesics (22\%), anti-depressants (18\%), 
methadone $(17 \%)$, hypnotics/sedatives $(15 \%)$; and cocaine $(11 \%)$. Interestingly, the jurisdictions in England and Wales reporting the highest annual drug-related death (DRD) rates per 100,000 population aged 16 years and over were not from metropolitan areas; i.e.: Blackpool \& the Fylde (19.4); Brighton \& Hove (17.8); Western Cumbria (17.3); and Newcastle-upon-Tyne (10.1). Conversely, coroners from more densely populated areas (i.e.: London, Liverpool, Birmingham and Manchester) reported much lower annual rates of DRDs.

Intriguingly, Dutch researchers in 1997 asked approximately 22,000 respondents, selected from large samples of the populations of four major cities and five categories of distinct address density municipalities throughout the Netherlands, about their lifestyles and use of licit and illicit drugs (21). The survey data revealed differences in terms of drug use prevalence in Amsterdam, Rotterdam, The Hague, and Utrecht, although the cities showed similar levels of address density. A distinct relationship was established between cannabis use and address density, with both lifetime and last-month prevalence rates being significantly higher in more densely populated municipalities. Similarly, cocaine prevalence rate was the highest in urbanized municipalities $(4.9 \%)$ and the lowest in rural municipalities (1.0\%). However, the 'experienced use' (i.e.: history of more than 25 occasions of cocaine intake) figures were not significantly related to the degree of urban residency. Conversely, ecstasy prevalence rates varied widely in the different address densities, with the lifetime prevalence rate for the high address density municipality being 3.6 percent, and 1.2 percent for the lowest address density municipalities. The age of first ecstasy use also appeared lower outside urban areas. Abraham (21) suggested that age may serve as a first explanatory variable for cannabis and ecstasy use, but not one for cocaine use (as there was no significant relationship between cocaine use and age in their study). In fact, many young people may move to urban areas to study at high schools or universities. Thus, the higher the address density of the municipality, the more young inhabitants there will be, and the higher the prevalence rates for substance use. In terms of prevalence rates, the cities of Rotterdam, The Hague and Utrecht were to be considered as one homogeneous group, and were quite distinct from Amsterdam.

Recently, Chen et al (22) focussed on substance use issues in China, where this represents a public health challenge. In fact, the rapidly growing rural-to-urban migrants, currently numbering 88 million, may be especially vulnerable to substance use. Levels of and risk factors for substance use were examined among 3,752 rural-to-urban migrants using cross-sectional data collected in 2002 in China. A moderation effect modelling analysis was applied to examine interactions of workplace with income and income with 
depression in predicting substance use. Respondents $(41.7 \%$ females) were 18 to 30 years of age with an average of 4.26 years of migratory experience. The 30-day alcohol intoxication rate was $16.8 \%$ for females and $36.7 \%$ for males; and lifetime use of illegal drugs was $1.8 \%$ for females and $1.6 \%$ for males. The Authors concluded that substance use was prevalent among rural-to-urban migrants, especially among female migrants, and that workplace, income, and depression were associated with substance use interactively.

In contrast with this, Klein and Pittman (23) examined whether region of residence may influence drinkers' alcohol consumption and/or their perceptions of alcohol use. Data were based on a national probability sample of American adults, of whom 1,069 were considered 'drinkers'. Results indicated that geographic region of residence made little difference in people's alcohol use and had only a limited impact on their drinking-related attitudes; they concluded that urbanization and gender are not influential variables in these relationships.

Urbanization, immigration, social norms and attitudes in promoting/mitigating substance misuse

Over the last few decades, people have increasingly moved from the countryside to the city or from a developing to a developed world city, making immigration primarily an urban phenomenon. Immigrants bring lifestyles (e.g. refusal to take intoxicants or alcohol) and support systems that may protect them against some of the adverse outcomes that other low-income urban residents experience, such as alcohol and drug misuse. Social norms and attitudes may reinforce healthy (or unhealthy) behaviours and contribute to better (or worse) physical and mental health in the index community. The importance of sociocultural factors in relation to race and gender in predicting onset and escalating of substance misuse was discussed by the important paper of Best et al (24), who examined stages of drinking and smoking careers and transitions from initiation to regular use among adolescents, as a function of ethnic status and gender. Data were collected from 1,777 adolescents, between the ages of 11 and 14, drawn from eight secondary schools in south-west London. For both smoking and drinking, white children were more likely to have ever smoked tobacco and drunk alcohol, and were also more likely to progress from initiation to regular use than were either black or Asian children. Asian children reported the latest onset and the lowest prevalence rates for both drinking and smoking. Males reported experimenting with both cigarettes and alcohol at an earlier age than females.

Gau et al (25) investigated the prevalence and changing trends of mental disorders and the effects of gender and urbanization among 
adolescents in Taiwan. They selected a random sample of seventhgrade students $(\mathrm{N}=1,070)$ from one urban and one rural junior high school in which $1,051(98.2 \%)$ and $1,035(96.7 \%)$ were reassessed in the second and third years, respectively. The most prevalent psychiatric condition was attention deficit hyperactivity disorder (ADHD) in the first 2 years and substance use disorders in the third. It emerged that rural adolescents had higher rates of conduct disorder, oppositional defiant disorder, and substance use disorders than their urban counterparts.

Fujiwara et al (26) aimed at determining the prevalence of drug abuse in a developing country undergoing rapid urbanization. They performed a household survey on the spread of drug abuse in Vientiane, Lao People's Democratic Republic and selected 1497 households from 17 villages through the stratified random sampling method from urban districts in the city of Vientiane. They inquired about the spread of drug abuse by asking if the families interviewed recognized drug abuse problems in their community. To examine the extent of urbanization of individual villages, an 'ad hoc' urban index was calculated by principle component analysis taking into account a number of indicators. Recognition of cases of drug abuse in the community and the urban index showed a significant correlation $(p<0.01)$. The Authors concluded that city neighbourhoods in a developing country undergoing rapid urbanization showed evidence of the spread of drug abuse, which was associated with the urban index.

Intra-urban differences and substance misuse levels

Intra-urban differences may be one of the key determinants of use of drugs in urban areas (1). In confirming this, Gemmell et al (27) aimed at establishing the prevalence of problem drug use in the 10 local authorities within the Metropolitan County of Greater Manchester between April 2000 and March 2001 using a multisample stratified capture-recapture analysis. The total number of problem drug users in Greater Manchester was estimated to be 19 255 giving a prevalence of problem drug use of 13.7 per 1000 population aged 16-54. The distribution of problem drug users varied across the 10 areas and they concluded that areas in close geographical proximity displayed different patterns of drug use in terms of prevalence rates and age and gender patterns.

Conversely, other studies have indeed not confirmed intra-urban disparities; comorbid severe mental illness and substance misuse (dual diagnosis) had been previously shown to be highly prevalent in inner-city populations. In taking into account these issues, Wright et al (28) identified representative prevalent cases with psychotic illnesses who had been in contact with services in a suburban area of South London over the previous 6 months; 61 of the 124 cases identified were randomly selected for interview, of whom $66 \%$ 
responded $(\mathrm{N}=40)$. The prevalence rates of dual diagnosis observed were $33 \%$ for any substance misuse, $20 \%$ for alcohol misuse only, $5 \%$ for drug misuse only, and $8 \%$ for both drug and alcohol misuse. Data showed that the prevalence of substance misuse in patients with severe mental disorders in this suburban area was about as high as that for similar patients in inner-city London.

Furthermore, Hay (29) aimed at estimating the prevalence of opiate or benzodiazepine misuse in the Grampian Health Board area, Scotland and illustrated the use of the capture-recapture method in both rural and urban settings. He identified a total of 1770 individuals as misusing opiates or benzodiazepines and residing in the area. The total number of opiate or benzodiazepine misusers in the City of Aberdeen was corresponding to $2.0 \%$ of the population aged 15-54 years. Conversely, in a smaller town to the north of Aberdeenshire (where high levels of heroin use had previously been reported in the media), $2.5 \%$ of the population aged $15-54$ were estimated to be misusing opiates or benzodiazepines.

\section{Discussion}

Although it has been suggested that the burden of substance misuse in large cities is greater than that in non-urban areas (2), this did not appear to be a consistent observation from the present literature review. In fact, the papers here identified that supported the existence of a causal relationship between urbanization and substance misuse $(7,18,21,22,26)$ were similar in number to both those that did not support said relationship $(6,20,23,25)$ and those which showed conflicting results $(8,10,19)$. One could then somehow conclude that the evidence about urban-rural differences in substance misuse is not clearly defined, probably because substance misuse behaviour is characterized by a multi-factorial aetiology. Intriguingly, there may be many positive and healthenhancing aspects which are characteristic of the urban context (30), including the relative proximity of health services and prompter emergency intervention in case of overdose. This is consistent with the observation that more densely populated UK areas typically report much lower annual rates of drug related deaths (20). Although Sundquist and Frank (18) concluded that high level of urbanization are associated with increased hospital admission rates for both alcohol and drug abuse, one may in fact need to take into account that hospitals are more easily reached, and admissions planned, when living in urban areas. In confirming this, Fortney et al (31) suggested that travel barriers significantly reduce treatment programme participation, especially for rural clients.

Indeed, the study of urban health must acknowledge the reality of complexity $(2 ; 21)$. In fact, the different cities may exhibit markedly 
different prevalence rates (21), and generalizing whole-population substance misuse figures may mask a number of differences that may exist between different population elements. Most papers here commented referred to substance use and misuse as a whole, using the term to reflect a broad range of behaviours including drug abuse, alcohol abuse, substance dependence and risky drug use behaviour (e.g. injection). However, this over-inclusive approach may offer a simplification of the complex relationship between the urban characteristics discussed here and the use and misuse of different substances. Only a few papers here commented considered if specific living conditions' features were differentially distributed between urban and nonurban areas and within urban areas. Furthermore, no studies quoted here empirically assessed a comprehensive, multi-factorial, model considering the contributions of the urban environment characteristics together with individuallevel determinants of substance use and misuse.

The present review paper may have some limitations. In fact, a manual search was not carried out to systematically look for papers published either in journals which are not covered in the Medline or as book chapters. Furthermore, a meta-analysis was not here carried out to either accept or reject the hypothesis of a causative association between urbanization and substance misuse. However, the range of papers identified was so diverse that this technique might not have been particularly useful.

Further research studies will need to be carried out to assess how different characteristics of the urban environment may be associated with substance-specific behaviours and their consequences. At a more general level, more efforts have to be put into explaining substance misuse in general. Identifying which characteristics of the urban context, and under which circumstances, are modifiable is an important theoretical, empirical, and public health question $(2 ; 32)$. 


\section{References}

Papers of particular interest have been highlighted as:

$*$ of special interest

** of outstanding interest

1. Galea S, Rudenstine S, Vlahov D. Drug use, misuse and the urban environment. Drug Alcohol Rev 2005; 24:127-136 (**) This is a very important paper, which offers one of the very few comprehensive overviews of the issues here discussed.

2. Galea S, Vlahov D. Urban health: evidence, challenges, and directions. Annu Rev Public Health 2005; 26:341-365.

3. Johnson TP, Fendrich M. Homelessness and drug use: evidence from a community sample. Am J Prev Med 2007; 32: S211-218 (*) An interesting paper, in which data are presented from a 2001 community survey in Chicago, that collected information regarding histories of drug use behaviour and homeless experiences from 627 young adults. Using covariance structure analyses, two alternative models of the association between drug use and homelessness were evaluated: social selection versus social adaptation processes.

4. Peen J, Dekker J, Schoevers RA, Have MT, de Graaf R, Beekman AT.Is the prevalence of psychiatric disorders associated with urbanization? Soc Psychiatry Psychiatr Epidemiol 2007; 42:984-9 (**) A convincing and well-designed study, which confirms that psychiatric disorders are more common and more complex in more urbanized areas.

5. Vlahov D, Galea S. Urbanization, urbanicity, and health. J Urban Health 2002; 79 (4 Suppl 1):S1-S12.

6. Jiang Z. Drug abuse among residents of Beijing: an epidemiologic survey of 1,822 households. Zhonghua Shen Jing Jing Shen Ke Za Zhi 1990; 23: 66-8, 125.

7. Joutsenniemi K, Martelin T, Kestilä L, Martikainen P, Pirkola S, Koskinen S. Living arrangements, heavy drinking and alcohol dependence. Alcohol Alcohol 2007; 42:480-91. (*) An interesting paper, aimed at assessing the variation in heavy drinking and alcohol dependence by living arrangements, and the contribution of social and behavioural factors to this variation

8. Madsen $\mathrm{MH}$, Grønbaek M, Bjerregaard P, Becker U; Greeland Population Study. Urbanization, migration and alcohol use in a population of Greenland Inuit. Int J Circumpolar Health 2005; 
9. March JC, Oviedo-Joekes E, Romero M. Drugs and social exclusion in ten European cities. Eur Addict Res 2006; 12:33-41

10. Day C, Conroy E, Lowe J, Page J, Dolan K. Patterns of drug use and associated harms among rural injecting drug users: comparisons with metropolitan injecting drug users. Aust J Rural Health 2006; 14:120-5.

11. Greacen T, Finkelstein C. Mental health care in Paris. Eur Psychiatry 2005; 20 Suppl 2:S285-8.

12. Squires NF, Beeching NJ, Schlecht BJ, Ruben SM. An estimate of the prevalence of drug misuse in Liverpool and a spatial analysis of known addiction. J Public Health Med 1995; 17:103-9.

13. Friedman SR, Tempalski B, Brady JE, Friedman JJ, Cooper HL, Flom PL, McGrath MM, Gostnell K, Des Jarlais DC. Predictors of the degree of drug treatment coverage for injection drug users in 94 metropolitan areas in the United States of America. Int J Drug Policy 2007; $18: 475-85$.

14. Littlejohn C, Baldacchino A, Schifano F, Deluca P. Internet pharmacies and online prescription drug sales: a cross-sectional study. Drug Educ Prevent Policy 2005; 12:75-80.

15. Schifano F, Deluca P, Agosti L, Martinotti G, Corkery JM and the Psychonaut 2002 research group: New trends in the cyber and street market of recreational drugs? The case of 2C-T-7 ("Blue Mystic"). J Psychopharmacol 2005; 19:675-679.

16. Deluca $P$, Schifano F. Searching the Internet for drug related websites; analysis of online available information on ecstasy (MDMA). Am J Addictions 2007; 16:479-83.

17. Mott J, Rathod $\mathrm{NH}$. Heroin misuse and delinquency in a new town. Br J Psychiatry 1976; 128:428-35

18. Sundquist K, Frank G.Urbanization and hospital admission rates for alcohol and drug abuse: a follow-up study of 4.5 million women and men in Sweden. Addiction 2004; 99: 1298-305.

19. Shah N, Landen MG. Unintentional deaths from drug poisoning by urbanization of area--New Mexico, 1994-2003. MMWR Morb Mortal Wkly Rep 2005; 54:870-3. 
20. Ghodse AH, Corkery J, Oyefeso A, Schifano F, Tonia T, Annan J. Drug related deaths in the UK. January - June 2006, np-SAD Surveillance Report No. 18. International Centre for Drug Policy, St George's, University of London (UK), 2007. (**) This is the annual National Programme on Substance Abuse Deaths (np-SAD), which provides the reader with accurate and updated figures of drug related fatalities across the UK.

21. Abraham M D. The impact of urban residency and lifestyle on illicit drug use in the Netherlands. J Drugs Issues 1999; summer issue: $565-586$

22. Chen, Xinguang, Stanton, Bonita, Li, Xiaoming, Fang, Xiaoyi, Lin, Danhua. Substance Use Among Rural-to-Urban Migrants in China: A Moderation Effect Model Analysis. Subst Use Misuse 2008; 43: 1082-1084. (*) This is an interesting study carried out in China, highlighting the substance misuse issues of those who migrate from rural areas.

23. Klein $\mathrm{H}$, Pittman DJ. Regional differences in alcohol consumption and drinkers' attitudes toward drinking. Am J Drug Alcohol Abuse 1993; 19:523-38.

24. Best D, Rawaf S, Rowley J, Floyd K, Manning V, Strang J. Ethnic and gender differences in drinking and smoking among London adolescents. Ethn Health 2001; 6:51-7.

25. Gau SS, Chong MY, Chen TH, Cheng AT. A 3-year panel study of mental disorders among adolescents in Taiwan. Am J Psychiatry 2005; 162: 1344-50.

26. Fujiwara T, Takano T, Nakamura $\mathrm{K}$. The spread of drug abuse in rapidly urbanizing communities in Vientiane, Lao People's Democratic Republic. Health Promot Int 2005; 20:61-8.

27. Gemmell I, Millar T, Hay G. Capture-recapture estimates of problem drug use and the use of simulation based confidence intervals in a stratified analysis. J Epidemiol Community Health 2004; 58:758-65.

28. Wright S, Gournay K, Glorney E, Thornicroft G. Dual diagnosis in the suburbs: prevalence, need, and in-patient service use. Soc Psychiatry Psychiatr Epidemiol 2000; 35:297-304.

29. Hay G. Capture-recapture estimates of drug misuse in urban and non-urban settings in the north east of Scotland. Addiction 2000; 95:1795-803. 
30. Newman P, Hogan T. A Review of urban density models: Toward a resolution of the conflict between populace and planner. Hum Ecology 1981; 9:269-303

31. Fortney JC, Booth BM, Blow FC, Bunn JY. The effects of travel barriers and age on the utilization of alcoholism treatment aftercare. Am J Drug Alcohol Abuse 1995; 21:391-406.

32. Galea S, Freudenberg N, Vlahov D. Cities and population health Soc Sci Medicine 2005; 60:1017-1033. (**) A seminal paper, which highlights the issues of urbanization and health. 